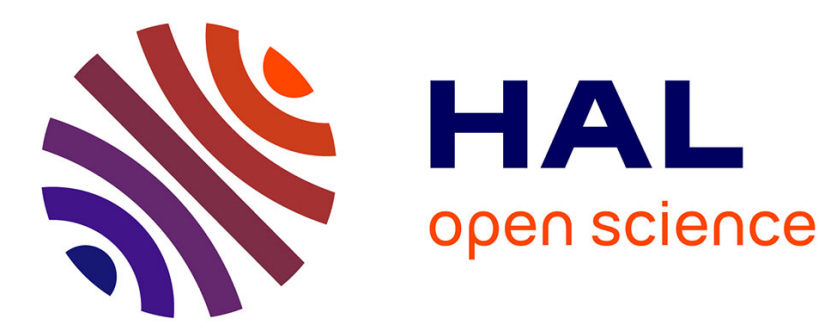

\title{
L'Inde : la loi avance, le patriarcat résiste
}

Kamala Marius

\section{To cite this version:}

Kamala Marius. L'Inde : la loi avance, le patriarcat résiste. Travail, genre et sociétés, 2017, 38, pp.193-199. 10.3917/tgs.038.0193 . halshs-02143355

\section{HAL Id: halshs-02143355 https://shs.hal.science/halshs-02143355}

Submitted on 29 May 2019

HAL is a multi-disciplinary open access archive for the deposit and dissemination of scientific research documents, whether they are published or not. The documents may come from teaching and research institutions in France or abroad, or from public or private research centers.
L'archive ouverte pluridisciplinaire HAL, est destinée au dépôt et à la diffusion de documents scientifiques de niveau recherche, publiés ou non, émanant des établissements d'enseignement et de recherche français ou étrangers, des laboratoires publics ou privés. 


\title{
L'Inde : la loi avance, le patriarcat résiste
}

\author{
Kamala Marius
}

Depuis son entrée en vigueur en 1950, la Constitution indienne est un document très favorable aux femmes puisque le droit de la discrimination fondée sur le sexe est garanti dans la liste des droits fondamentaux justiciables. Au-delà de l'égalité des sexes, elle assure dans son article 14, que «le gouvernement ne peut dénier à une personne le droit à l'égalité devant la loi ou une égalité de protection ». L'article 15 de la Constitution indienne déclare que «le gouvernement ne peut discriminer aucun citoyen sur la base du sexe seulement ». L'article 16, enfin, garantit qu' « aucun citoyen ne pourra faire l'objet de traitement discriminatoire en matière d'emplois publics sur la base du sexe seulement ». On pourrait donc penser que la société indienne est entrée dans une ère de post patriarcat.

Cependant, en dépit d'une constitution indienne égalitaire, d'une présence de plus en plus importante des femmes dans le domaine politique et économique, la société demeure patriarcale. Comment l'expliquer?

\section{La famille : socle de la société patriarcale}

La famille élargie ou indivise (joint family) a longtemps fondé l'unité même du système social indien, plus à la campagne qu'à la ville. En effet, ce sont les normes et pratiques familiales patriarcales qui confèrent aux individus et aux femmes en particulier, leur identité et leurs poids relatifs dans la parenté et la communauté. La parenté est là pour définir notamment le rôle des fils et des filles, des femmes et des mères. Elle leur assigne des fonctions spécifiques selon quelques principes simples : l'appartenance de caste ou de clan, l'âge, le rang d'aînée, le statut matrimonial et familial, et surtout le genre. Dès quinze ans, les garçons se voient reconnaître le statut d'adultes à la différence des filles. 
Ce patriarcat impose une dichotomie spatiale symbolique entre masculin et féminin en confinant les femmes à des espaces assignés en fonction des hommes, sachant que les frontières de ces territoires restent poreuses et constamment qualifiées et requalifiées. Il est encore d'usage par exemple, dans certaines castes chez les hindous et les musulmans, que les femmes de la maison servent le repas aux hommes avant de prendre le leur dans la cuisine. En revanche, chez les chrétiens qui ont adopté les pratiques hindoues par mimétisme, cela tend rapidement à disparaître. En règle générale, les femmes de haute caste sont soumises à un confinement spatial plus important que les autres, d'où une situation de domination même si elle n'est pas consciente. Des variations sont à apporter selon que les femmes sont mariées ou pas : en effet le mariage les assigne plus facilement à la maison et les expose moins à l'extérieur dès lors qu'elles s'occupent des enfants. Quant aux femmes non mariées, en raison de la réputation de la famille et de leur soi-disant vulnérabilité, elles sont contraintes de sortir accompagnées.

Dans ce système patriarcal, les femmes sont plutôt valorisées comme mères de garçons, d'autant que ces garçons sont appelés à prolonger les lignées familiales. Ainsi, des pratiques comme l'infanticide féminin visent justement à favoriser la masculinité de la descendance. Certains y ont vu aussi la prégnance des structures communautaires patrilinéaires tant chez les hindous que chez les musulmans mettant en avant «l'honneur» (izzat) dans le cadre de khap panchayat (conseils masculins de villages traditionnels illégaux). En effet on a pu observer une surmasculinisation chez les hindous et les sikhs Jats (castes dominantes) du Penjab et de l'Haryana ou encore dans les milieux conservateurs hindous et musulmans du Gujarat et du Rajasthan, qui pratiquent les crimes d'honneur et l'infanticide selon la «coutume (rivaj, parampara). Être une femme en Inde implique souvent une part importante de déni, voire de haine de soi. » [Heuzé, 2014, p. 277].

En revanche, tout homme se souvient toujours avec nostalgie de son enfance avec ses grands-parents, oncles, cousins... En effet, bon nombre d'Indiens passent les années déterminantes de la prime enfance dans un contexte familial 
communautaire. La réalité de la famille élargie est si forte que la séparation d'avec elle, si elle doit avoir lieu, se fait le plus tard possible, notamment pour les fils dont les liens avec la mère sont si forts depuis la naissance qu'ils occultent toute notion d'indépendance et d'autonomie pendant l'enfance. Si les femmes ne «sentimentalisent » pas la relation à leurs mères, les hommes en revanche ont du mal à se séparer psychologiquement d'elle. Cette proximité envahissante de la mère et « cette subjugation maternelle » inconsciente [Kakar et Kakar, 2007, p. 129] favorisent peu les relations intimes avec l'épouse et sont à l'origine de nombreux conflits entre la bru et la belle-mère. Le rôle puissant que joue la déesse mère (notamment les mères sur leur fils) dans l'imaginaire culturel indien limite tout signe d'attachement et de tendresse de la part de ces maris qui restent sous la coupe de leur mère très possessive. Ainsi, rares sont les moments d'intimité dans la famille indivise.

En fait, les relations familiales sont gérées sur la base d'un principe hiérarchique séculaire. Toute personne doit respect et obéissance au chef de famille qui est généralement le père ou l'homme le plus âgé de la communauté familiale. Les principes hiérarchisants de ce système sont l'âge et le sexe. Les aînés jouissent d'une autorité incontestée et plus importante que les cadets. Certes, les hommes ont plus d'autorité que les femmes, mais les femmes mariées les plus âgées ont une autorité importante au sein de la famille.

Ainsi, quel que soit son statut économique et social, la position d'un homme ou d'une femme dans la hiérarchie familiale est définitive : ses obligations envers ses « supérieurs » et ses exigences envers ses « subordonnés » sont immuables. En tant que futur chef de famille, le fils aîné jouit d'une position presque aussi puissante que le père ; ses cadets, leurs femmes et leurs enfants lui doivent respect. Le fils aîné est donc le pivot central de la famille car il assure la prise en charge de la vieillesse de ses parents, reprend la terre, perpétue la lignée et surtout accomplit les rites funéraires, d'où l'obstination des hindous à avoir au moins un fils. Une fille ne peut pas conduire les rituels, ne peut pas hériter selon les traditions - mais, elle le peut selon la loi - car la famille lui fournit une dot à son mariage.

L'Inde : la loi avance, le patriarcat résiste. Kamala Marius 


\section{Le mariage : une institution patriarcale}

Destiné à perpétuer la famille quelle que soit la caste ou la communauté, le mariage est avant tout un arrangement conclu entre les parents qui ont le devoir formel de marier leurs enfants. Si, depuis l'Antiquité, de nombreuses formes de mariage ont existé, du don au rapt de la très jeune fille, à l'heure actuelle la pratique la plus courante, grâce aux sites de rencontres sur Internet, consiste en un accord entre les deux familles de même caste, de même religion et de même rang social, voire de même profession si possible. La virginité est exigée, d'où le développement des industries de reconstruction d'hymen. Dans le monde hindou des hautes castes qui joue le rôle de modèle normatif, le divorce est déshonorant, même s'il est de plus en plus fréquent. Les femmes qui ont des amants veillent à ne pas franchir certaines limites [Heuzé, 2014].

«La pérennité du consensus en faveur du mariage arrangé est stupéfiante » [Kakar et Kakar, 2007, p. 83]. Ce consensus est fondé sur l'acceptation de la définition culturelle $\mathrm{du}$ mariage comme une affaire familiale plus qu'individuelle, d'autant que le mariage d'amour est souvent réputé mal finir en dépit de la popularité des films Bollywood qui font rêver en racontant de telles histoires. Bon nombre d'enquêtes réalisées en milieu rural tamoul révèlent le peu d'exigence de la part des femmes ; l'essentiel, disent-elles, «c'est qu'il ne boive pas, qu'il ne me frappe pas et qu'il subvienne aux besoins de la famille ». À l'exception de quelques jeunes gens des campus universitaires ou de citadins très occidentalisés qui refusent le système en général, la coutume de la dot (dowry) interdite par la loi est répandue dans l'Inde entière, quelles que soient la caste, la classe sociale et la religion (sikhs, chrétiens et musulmans compris).

Inutile d'évoquer la tyrannie exercée par la belle-famille sur les brus fournisseuses de dots, phénomène bien connu grâce aux médias. Si par malheur, la belle-famille n'a pas fini de verser la dot promise, la famille du mari n'hésite pas à assassiner la nouvelle bru, maquillant le crime en suicide. Pendant longtemps, les coupables n'étaient jamais poursuivis, mais devant l'ampleur du phénomène et grâce au fameux article du code pénal 498a adopté en 1983, des 
procès commencent à être intentés avec succès (15\% en 2012), malgré des mouvements masculinistes liés au révisionnisme ambiant (en lien avec l'arrivée au pouvoir du gouvernement nationaliste hindou). Cette pratique de la dot est la principale cause d'endettement des familles indiennes et, malgré le niveau d'éducation de plus en plus élevé des jeunes filles, elle n'est pas près de disparaître.

L'idéal de la parfaite épouse que l'on retrouve dans la mythologie, malgré les conséquences que peuvent avoir les progrès de l'éducation, de l'urbanisation et de la modernisation, se traduit non seulement par un dévouement exclusif au mari et, dans une moindre mesure, aux parents de celui-ci, mais aussi par le désir de donner naissance à des enfants mâles.

\section{Des lois communautaires patriarcales}

Certes, la dot, la fécondité sélective, le harcèlement sur le lieu de travail, le viol sont punis par la loi. Le domestic violence act définit la violence domestique comme une violation des droits humains. Mais les procédures continuent d'être longues car elles sont biaisées par des préjugés de genre.

Dans le cas de la loi sur les mariages de 1956 (Special Marriage Act), les hommes et les femmes bénéficiaient des mêmes droits au divorce, basés sur le constat de cruauté. Cependant, dans la pratique, les tribunaux ne faisaient rien pour modifier le modèle patriarcal de « la bonne épouse ».

En effet, la représentation des femmes comme épouses et mères, faibles, passives et nécessitant protection, inspire largement les interprétations et décisions judiciaires. Finalement la famille reste construite comme une sphère privée, échappant à l'intervention légitime de la loi. Même les interprétations judiciaires des lois sur le viol, la dot, les crimes d'honneur continuent d'être nourries par une idéologie «familialiste » et ont finalement pour effet de renforcer le contrat moral qui pèse sur les femmes. Agnès Flavia montre bien «dans quelle mesure les mêmes vieilles notions de chasteté, de virginité, de 
prime au mariage et de peur de la sexualité féminine se reflètent dans les jugements fondés sur la nouvelle loi sur le viol, telle qu'amendée » [Flavia, 1992, p. 21]. Dans le cas du viol, par exemple, une femme peut voir aboutir ses revendications si elle est prête à se présenter comme chaste, pure, virginale et modeste.

En matière d'affaires familiales, l'Inde ne possède pas un code civil unique qui lui permettrait de légiférer de manière identique dans tout le pays. Bien que l'uniformisation du Code civil ait été prévue par la Constitution, peu d'efforts ont été accomplis en ce sens. En effet, pour tout ce qui relève des lois sur la famille, le mariage, le divorce, la pension alimentaire, l'adoption, l'héritage et succession, c'est la loi dite personnelle qui prévaut. Ce terme « personnel » a été introduit dans la sphère légale pour conserver la distinction entre espace public et privé, de peur qu'une uniformisation des lois régissant la sphère privée entraîne des conflits religieux. La liberté de réguler la sphère domestique en accord avec ses convictions religieuses a été offerte aux chefs religieux et aux minorités, en contrepartie de l'acceptation d'une politique uniforme du droit public. Ainsi, les lois régissant le mariage, le divorce, les successions et l'adoption, soit tout le quotidien de l'individu, ont été promulguées dans différents codes religieux par le gouvernement britannique ${ }^{1}$, puis par les gouvernements indiens successifs ${ }^{2}$, dans le but d'éviter des conflits religieux et de stabiliser leur pouvoir en écartant par ce biais la question sensible des minorités.

Le système légal en Inde doit encore dénouer cette contradiction fondamentale entre l'autonomie des femmes, rendue possible grâce aux différentes législations

1 Tout avait commencé par l'abolition du rituel du Sati en 1829, mais cela ne concernait que les femmes de religion hindoue.

2 Elisabeth Moretti- Rollinde, «La question des "droits personnels" en Inde en l'absence d'un code civil unique », Bulletin du Credho, n 14 - décembre 2004. 
progressistes, et leur traitement concret par le droit personnel et coutumier de leurs communautés.

Des réformes des codes du statut personnel s'imposent mais elles doivent prendre en compte les identités à l'intérieur des frontières religieuses : très peu d'attention a été conférée aux multiples identités transversales différenciant les femmes musulmanes ou hindoues entre elles, en fonction de l'appartenance de caste, de classe, de la langue ou de la région.

\section{Pour une approche intersectionnée des sociétés}

\section{patriarcales}

Cela fait écho à l'analyse fondatrice de l'intersectionnalité qui met en évidence l'incapacité des politiques publiques indiennes à prendre en compte le caractère multidimensionnel de la subordination à laquelle les femmes pauvres de basses castes sont soumises.

Ce paradigme de l'intersectionnalité dans un contexte de domination, propose de penser ensemble les rapports sociaux de genre, de caste, de classe, de communauté, partant du principe que les principaux systèmes d'oppression sont interlocked (inter-reliés). Cette contribution théorique transdisciplinaire majeure du black feminism vise à appréhender la complexe articulation des identités et des inégalités multiples par une «approche intégrée ». Elle réfute le cloisonnement et la hiérarchisation des grands axes de la différenciation sociale que sont les catégories de sexe/genre, classe, race, [Crenshaw 1993 ; Hill Collins 1990, 2000]. «Le paradigme intersectionnel, bien loin de dévoyer ou de diluer le féminisme (au motif que la race et la classe affaibliraient son message), permet au contraire, dans chaque situation particulière, dans chaque contexte, de préciser les différents paramètres de la domination, notamment la domination liée au rapport social de sexe, en regardant systématiquement l'intrication des rapports sociaux. » [Lénel et Martin, 2012, p. 141]. 
Dans la mouvance des théories féministes postcoloniales rejetant l'hégémonisme occidental comme entreprise de manipulation des féminismes locaux, un féminisme indigène ${ }^{3}$ indien s'est développé dans les années 1990. Il s'oppose à un féminisme de hautes castes, élitaire et urbain. En effet, ce débat est récurrent, au sein du mouvement féministe, à l'occasion du débat annuel sur le Women's Reservation Bill, puisque l'opposition la plus virulente à ce projet de loi émane des partis représentant les intouchables, les basses castes et les musulmans. Tous ces groupes y voient un complot des élites, visant, sous couvert d'améliorer la représentation électorale des femmes, à rendre aux castes supérieures la place qu'elles ont perdue, au profit des basses castes, dans les assemblées législatives.

En fait, les relations de pouvoir ne peuvent être modifiées, qu'en agissant sur trois fronts : en remettant en cause l'idéologie qui justifie les inégalités (comme les rapports de genre ou la caste); en changeant les modalités d'accès et de contrôle des ressources économiques, naturelles et intellectuelles; en transformant les institutions et les structures qui renforcent et maintiennent les rapports de pouvoir existants (tels que la famille, l'État, le marché, l'éducation ou les médias) [Batliwala, 1993].

Cette réflexion sur l'intersectionnalité des catégories de genre, race, communauté et caste a très largement nourri le champ des postocolonial studies, des diaspora studies, des queer studies, mais reste peu mobilisée en France. Ainsi on pourrait dire que la société indienne, plutôt que de se trouver dans une situation de post patriarcat, se situe dans un contexte patriarcal postcolonial. Les questions de genre imposent d'orienter le regard sociétal autrement, en tenant compte des discours et des représentations, pour mieux analyser les mécanismes d'oppression et de domination qui sont insuffisamment interpellés pour le moment.

3 L'anthologie passionnante, éditée par Maitrayee Chaudhuri, Feminism in India, en 2004 , consacre toute une section à ce féminisme indigène. 


\section{Bibliographie}

Batliwala Srilatha, 1993, Women's Empowerment in South Asia: Concepts and Practices, New Delhi, aspbae/fao.

Crenshaw Kimberlé Williams, 1993, «Mapping the Margins: Intersectionality, Identity Politics, and Violence against Women of Color », Stanford law review, vol. 43, pp. 1240-1299.

Dorlin Elsa, 2009, Sexe, race, classe: pour une épistémologie de la domination, Paris, puf, coll. «Actuel Marx ».

Flavia Agnès, 1992, «Protecting Women against Violence? Review of a Decade of Legislation, 1980-89 », Economic and Political Weekly, 27 (17), pp. 19-33.

Heuzé Djalal, 2014, «Un viol en Inde », Les Temps modernes, 2014/2, $\mathrm{n}^{\circ}$ 678, pp. 266-279.

Hill Collins Patricia, 2000 [1990], Black Feminist Thought: Knowledge, Consciousness and the Politics of Empowerment, Oxford, Routledge.

Kakar Sudhir et Kakar Katharina, 2007, Les Indiens, portrait d'un peuple, Paris, Seuil.

Lénel Pierre et Martin Virginie, 2012, «La contribution des études postcoloniales et des féminismes du Sud à la constitution d'un féminisme renouvelé. Vers la fin de l'occidentalisme ? », Revue du Tiers Monde, $\mathrm{n}^{\circ}$ 209, pp. 125-144.

Marius Kamala, 2016, Les inégalités de genre en Inde au prisme des études féministes postcoloniales, Paris, Karthala.

Spivak Gayatri, 2009, Les subalternes peuvent-elles parler?, Paris, éd. Amsterdam. 